\title{
Iluminação de Ambientes Tridimensionais Virtuais com Traçado de Raios \\ Virtual Three-Dimensional Environment Illumination With Tracing Of Rays
}

\author{
Jason Paulo Tavares Faria Junior ${ }^{1}$
}

\begin{abstract}
Resumo
Neste trabalho será desenvolvido um software que permite a visualização tridimensional de ambientes externos e internos utilizando para iluminação destes cenários a técnica Ray Tracing (traçado de raios), envolvendo várias disciplinas como computação gráfica, arquitetura, eletromagnetismo, matemática, topografia, linguagem de programação e física. Este projeto é dirigido aos alunos dos últimos anos de graduação e de iniciação científica com o objetivo de desenvolver este procedimento automatizado com a finalidade de comercialização.
\end{abstract}

Palavras chave: Visualização Tridimensional; Iluminação; Traçado de raios

\begin{abstract}
In this work will be developed a software that consents to a three-dimensional visualization of an external and internal environment using for illumination of these scenes the technique Ray Tracing (tracing of rays). Involving several disciplines as graphical computation, architecture, electromagnetism, mathematics, topography, programming language and physics. This project is guided to the pupils of the last years of graduation and scientific initiation with the objective to develop this automatized procedure with the commercialization purpose.
\end{abstract}

Keywords: Three-dimensional Visualization; Illumination; Tracing of Rays. 


\section{Introdução}

Um dos objetivos da realidade virtual é possibilitar a realização de experiências, treinamentos e o desenvolvimento de habilidades que sejam transferíveis para o mundo real. $\mathrm{O}$ uso de ambientes com alto grau de realismo desempenha um papel importante, tornando os ambientes virtuais mais próximos da experiência diária de seus usuários.

Com o trabalho de Keller (Teoria Geométrica da Difração -TGD, 1962), a técnica Ray Tracing (traçado de raios) recebeu um grande impulso e recentemente também tem sido utilizada para simular o efeito da iluminação em cenários tridimensionais no computador e na área de telecomunicações para caracterização da propagação da energia em áreas urbanas visando determinar a atenuação do sinal de celulares neste tipo de ambiente, pois a luz do sol ou de uma lâmpada ou mesmo os sinais de radiodifusão (celular, televisão, FM, AM, etc.) são ondas eletromagnéticas e podem ser simuladas através do Ray Tracing. Duas técnicas distintas para a elaboração do algoritmo de traçado de raios são citadas na literatura: o traçado baseado em imagens (Faria,2000) e o traçado por "força-bruta" (Rodrigues,1990). A filosofia de ambas é calcular a contribuição de todos os raios emitidos pela fonte (Sol, lâmpada ou transmissor) e que chegam a um objeto (receptor) após determinado número de difrações e reflexões nas paredes dos obstáculos.

Em 1994, pesquisadores de Barcelona (Espanha) desenvolveram um programa (Ruiz-Boqué S. e outros, 1994) baseado em traçado de raios aplicável a grades urbanas 3D genéricas, sobre terrenos planos. O programa opera sobre mapas digitalizados e associa vetores $2 \mathrm{D}$ a cada edifício ou quarteirão. As demais características individualizadas tais como, altura, permissividade, condutividade e rugosidade são incorporadas ao arquivo que contém o mapa, através de rotinas específicas do programa, escrito em linguagem C. O modelo considera os mecanismos de propagação por iluminação direta (visada direta), difração e a reflexão nas fachadas das edificações e no solo. A reflexão é calculada com base nos coeficientes de Fresnel. A difração, por sua vez, é computada com base na Teoria Uniforme da Difração (TUD) da Física Eletromagnética.

O uso de iluminação no processo de geração de cenários no computador contribui significativa- mente para o aumento do grau de realismo em imagens sintetizadas por meio de técnicas de computação gráfica. Esse processo, por sua vez, não é uma tarefa trivial, devendo considerar simultaneamente variações nas dimensões espacial e direcional da iluminação de uma cena. Os objetos que se deseja adicionar no cenário podem ser simulados por uma ferramenta de modelagem tridimensional (3D). Essa iluminação é feita de forma que cada pixel da imagem seja influenciado pelo feixe de luz adequado como se os objetos estivessem presentes em um cenário real. Para garantir um alto grau de realismo, ainda poderá ser necessário adicionar sombras ou reflexos projetados pelos objetos. A Figura 1 ilustra os resultados obtidos com este processo. Atualmente, existem diversos algoritmos de iluminação de superfícies e sólidos, porém nenhum deles é tão poderoso quanto o Ray Tracing.

\section{Materiais e Métodos}

Inicialmente será realizada a etapa de análise em que se verifica a melhor maneira de executar a tarefa e que recursos serão necessários como, por exemplo: números de programadores, linguagem de programação e hardware. Na próxima fase, chamada de Projeto, determina-se os "Lay-outs" das telas e características do software. Posteriormente insere-se o código do software na linguagem de programação determinada na fase de análise. Em seguida, realizamse os testes no produto para verificar se os requisitos concordados na especificação (Fase de projeto) estão sendo atendidos. Quando o procedimento automatizado estiver pronto, inicia-se a fase de manutenção.

O programa que será desenvolvido neste trabalho implementará a técnica de Ray Tracing em cenários tridimensionais. A interface gráfica para o usuário será projetada para operar com tela de apresentação, caixas de diálogo e interfaces de visualização dos cenários.

Os mapas digitalmente obtidos poderão ser carregados no programa através de um arquivo de extensão .BMP, .WMF ou .ICO (Figura 2). Esses formatos permitem que ambientes reais gerados em digitalização de mapas e "softwares" comerciais, sejam transportados para o programa.

No interior das edificações, após a visualização do cenário tridimensional (Figura 1), serão aplicados os algoritmos de Ray Tracing, sendo que, os 
posicionamentos das fontes luminosos (lâmpadas ou sol) deverão ser escolhidos pelo usuário.

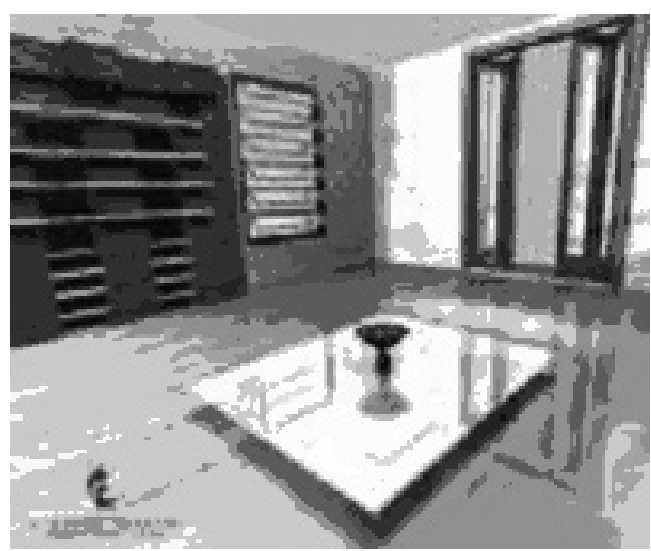

Figura 1- Visualização do cenário tridimensional

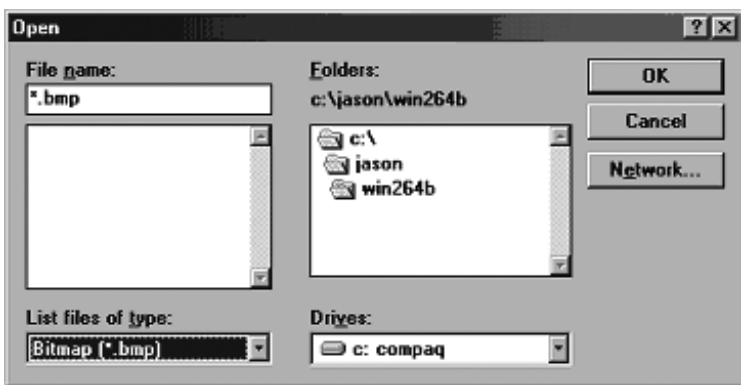

Figura 2- Exemplo de caixa de diálogo para a inserção dos mapas

Áreas Urbanas também serão visualizadas. A tela de simulação também possuirá uma barra de menus, onde o usuário tem acesso aos formulários de entrada de dados e visualização dos gráficos.

\section{Descrição do programa de traçado de raios}

Para o cálculo do nível de iluminação recebida, o programa inicia com o traçado de um raio emitido pelo transmissor, em uma direção específica. $\mathrm{O}$ lançamento de raios é definido por incrementos de q e f em coordenadas esféricas centradas na fonte, conforme a descrição da figuras 3 e 4 .

Nos obstáculos, os raios são refletidos ou difratados. Cada raio é traçado até as condições de descarte de raios forem atingidas.

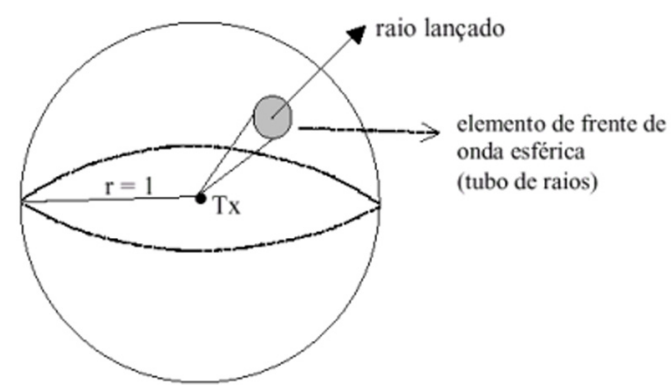

Figura 3- Lançamento de raios em um ambiente tridimensional.

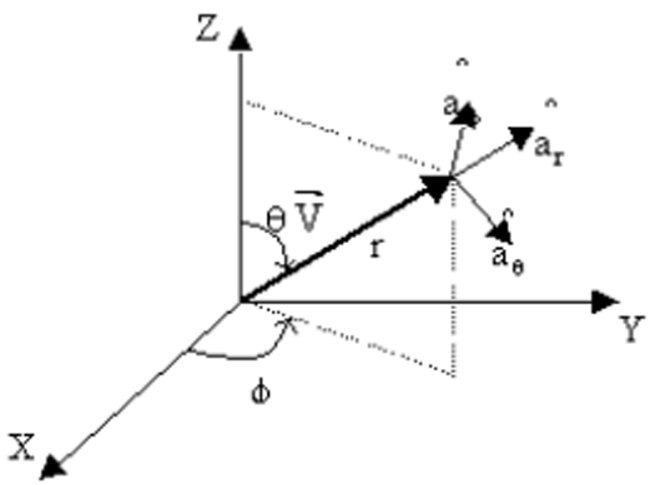

Figura 4- Coordenadas esféricas para o lançamento de raios.

A amplitude do sinal para cada raio atenua reciprocamente com a distância e por perdas adicionais provocadas por reflexão e difração no ambiente.

A expressão a seguir fornece o campo elétrico de um raio direto em uma posição s qualquer.

$$
\overrightarrow{\mathrm{E}}(\mathrm{s})=\overrightarrow{\mathrm{E}}_{d} \frac{\mathrm{e}^{-j \beta s}}{s}
$$

Sendo:

$$
\overrightarrow{\mathrm{E}_{d}}(\theta, \phi)=\sqrt{\frac{\eta \mathrm{P}_{\mathrm{t}} \mathrm{G}_{\mathrm{t}}}{2 \pi}} \overrightarrow{\mathrm{E}_{0}}(\theta, \phi)
$$

onde:

$\overrightarrow{\mathrm{E}_{0}}(\theta, \phi)$ - diagrama de irradiação da fonte transmissora, normalizado.

$\mathrm{G}_{\mathrm{t}}$ - ganho da fonte transmissora

$\eta$ - impedância do espaço livre: $\eta=120 \pi[\Omega]$

$\mathrm{P}_{\mathrm{t}}$ - potência de transmissão [W]

com, $\beta=2 \pi / \lambda$

\section{Cálculo dos campos refletido e difratado}

O ponto de intercessão do raio com algum plano (parede ou solo) é calculado com base no ponto de origem do raio, seu ângulo de lançamento com 
relação a um eixo de coordenadas $\mathrm{x}$, y e z, e as coordenadas do plano.

Testes são realizados a cada incremento que o raio sofre ao se propagar, tornando-o capaz de identificar se o raio atingiu um plano (reflexão), quina (difração) ou atingiu as paredes externas da construção na qual se deseja fazer a predição. Se o raio atingiu estas paredes, sub-programas determinam as distâncias de propagação dos raios, o número de reflexões, o ângulo de difração e os ângulos de reflexão. O processo se reinicia com um novo traçado de raio a partir do transmissor, com um ângulo de incrłmento o em relação ao raio inicial. O processo se repete até que todas as direções de lançamento a partir da fonte sejam computadas.

Para campos refletidos, a aplicação dos sistemas de coordenada fixo ao raio para a onda refletida é demonstrada na figura 5. Um raio emitido de uma fonte $\mathrm{S}$ intercepta um obstáculo na posição $\mathrm{R}$, onde este é refletido. A posição $\mathrm{F}$ é o ponto de observação ao longo do raio refletido. Os comprimentos dos segmentos SR e RF são denotados por $\mathrm{S}_{1}$ e $\mathrm{S}_{2}$, respectivamente.

$\mathrm{O}$ campo refletido $\mathrm{E}_{\mathrm{R}}^{\mathrm{T}}(\mathrm{x}, \mathrm{y}, \mathrm{z})$ na posição $\mathrm{R}$ é determinado pelo campo incidente $\mathrm{E}_{\mathrm{R}}^{\mathrm{i}}(\overline{\mathrm{x}}, \mathrm{y}, \mathrm{z})$ na posição R pela relação

$\overline{\mathrm{E}}_{\mathrm{R}}^{\mathrm{r}}(\mathrm{x}, \mathrm{y}, \mathrm{z})=\overline{\overline{\mathrm{R}}} \overline{\mathrm{E}}_{\mathrm{r}}^{\mathrm{i}}(\mathrm{x}, \mathrm{y}, \mathrm{z})$

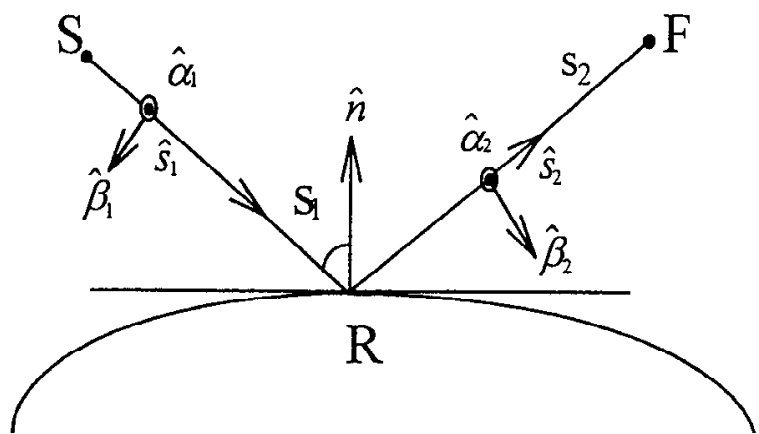

Figura 5- Sistema de coordenada de raio fixo numa interface.

Onde $\overline{\overline{\mathrm{R}}}$ é chamado de matriz de reflexão, com dimensão $3 \times 3$ em um sistema de coordenada fixo ao cenário $(\mathrm{x}, \mathrm{y}, \mathrm{z})$. As coordenadas incidentes e refletidas fixo ao raio, representadas por $\left(\hat{s}_{1}, \hat{\alpha}_{l}, \hat{\beta}_{I}\right)$ e $\left(\hat{s}_{2}, \hat{\alpha}_{2}, \hat{\beta}_{2}\right)$, respectivamente, são introduzidos para reduzir o número de elementos da matriz. $\hat{s}_{1}$ é o vetor unitário incidente ao longo da direção entre o ponto fonte $\mathrm{S}$ para o ponto de reflexão R. $\hat{s}_{2}$ é o vetor unitário ao longo da direção entre o ponto $\mathrm{R}$ para o ponto F. $\hat{n}$ é o vetor normal unitário do obstáculo. Define-se $\hat{\alpha}_{i}$ e $\hat{\beta}_{i}, i=1,2$, por:

$$
\begin{array}{ll}
\hat{\alpha}_{1}=\frac{\hat{s}_{1} x \hat{n}}{\left|\hat{s}_{1} x \hat{n}\right|} & \hat{\alpha}_{2}=\frac{\hat{s}_{2} x \hat{n}}{\left|\hat{s}_{2} x \hat{n}\right|} \\
\hat{\beta}_{1}=\frac{\hat{s}_{1} x \hat{n}}{\left|\hat{s}_{1} x \hat{n}\right|} & \hat{\beta}_{2}=\frac{\hat{s}_{2} x \hat{n}}{\left|\hat{s}_{2} x \hat{n}\right|}
\end{array}
$$

Os vetores $\hat{\beta}_{1}$ e $\hat{\beta}_{2}$ são vetores nos planos incidente e refletido, no sistema de coordenadas $\left(\hat{s}_{1}, \hat{\alpha}_{1}, \hat{\beta}_{1}\right)$ e $\left(\hat{s}_{2}, \hat{\alpha}_{2}, \hat{\beta}_{2}\right), \bar{E}_{r}^{i}(\mathrm{x}, \mathrm{y}, \mathrm{z})$ e $\bar{E}_{R}^{r}(\mathrm{x}, \mathrm{y}, \mathrm{z})$ são expressadas por $\left(E_{\alpha 1}^{i}, E_{\beta 1}^{i}, E_{s 1}^{i}\right)$ e $\left(E_{\alpha 2}^{i}, E_{\beta 2}^{i}, E_{s 2}^{i}\right)$, respectivamente.

Com este novo sistema de coordenadas, a componente das ondas refletidas na direção do raio torna-se zero. Então $E_{s 1}^{i}=E_{s 2}^{r}=0$. Logo, obtem-se :

$\bar{E}_{R}^{i}(x, y, z)=\alpha_{1} E_{\alpha 1}^{i}+\beta_{1} E_{\beta 1}^{i}$

$\bar{E}_{R}^{r}(x, y, z)=\alpha_{2} E_{\alpha 2}^{i}+\beta_{2} E_{\beta 2}^{i}$

$\overline{\overline{\mathrm{R}}}=\left(\begin{array}{ll}\mathrm{R}_{\alpha \alpha} & \mathrm{R}_{\alpha \beta} \\ \mathrm{R}_{\beta \alpha} & \mathrm{R}_{\beta \beta}\end{array}\right)$

Onde $\mathrm{R}_{\alpha \alpha}=\mathrm{R}_{\mathrm{s}}, \mathrm{R}_{\beta \beta}=\mathrm{R}_{\mathrm{p}}$ e $\mathrm{R}_{\alpha \beta}=\mathrm{R}_{\beta \alpha}=0$

Coeficientes de reflexão de Fresnel para polarização perpendicular (horizontal) e paralela (vertical) são dadas por

$$
\begin{aligned}
& \mathrm{R}_{\mathrm{H}}=\mathrm{R}_{\mathrm{p}}=\frac{\mathrm{n}_{2} \cos \left(\theta_{\mathrm{i}}\right)-\mathrm{n}_{1} \cos \left(\theta_{\mathrm{t}}\right)}{\mathrm{n}_{2} \cos \left(\theta_{\mathrm{i}}\right)+\mathrm{n}_{1} \cos \left(\theta_{\mathrm{t}}\right)} \\
& \mathrm{R}_{\mathrm{V}}=\mathrm{R}_{\mathrm{s}}=\frac{-\mathrm{n}_{1} \cos \left(\theta_{\mathrm{i}}\right)+\mathrm{n}_{2} \cos \left(\theta_{\mathrm{t}}\right)}{\mathrm{n}_{1} \cos \left(\theta_{\mathrm{i}}\right)+\mathrm{n}_{2} \cos \left(\theta_{\mathrm{t}}\right)}
\end{aligned}
$$

Onde $n_{1}$ e $n_{2}$ são as impedâncias intrínsecas do meio um e dois respectivamente. Sendo que:

$$
n_{m}=\sqrt{\frac{j w \mu_{m}}{\tau_{m}+j w \varepsilon_{m}}}
$$

A permissividade ou constante elétrica $\left(\varepsilon_{\mathrm{m}}\right)$, a permissividade $\left(\mu_{m}\right)$ e a condutividade $\left(\tau_{m}\right)$ caracterizam as propriedades de cada meio na interface, e w é a freqüência angular.

Considerando o meio 1 como sendo o $\operatorname{ar}\left(\varepsilon_{\mathrm{r}}=\varepsilon_{0}\right)$ e a permeabilidade do meio 2 igual à do vácuo, as expressões 
dos coeficientes de reflexão, tornam-se mais simples, ou seja :

$$
\begin{aligned}
& R_{v}=\frac{\cos \left(\theta_{i}\right)-\sqrt{\varepsilon_{r}-\operatorname{sen}^{2}\left(\theta_{i}\right)}}{\cos \left(\theta_{i}\right)+\sqrt{\varepsilon_{r}-\operatorname{sen}^{2}\left(\theta_{i}\right)}} \\
& R_{H}=\frac{\bar{\varepsilon}_{r} \cos \left(\theta_{i}\right)-\sqrt{\varepsilon_{r}-\operatorname{sen}^{2}\left(\theta_{i}\right)}}{\bar{\varepsilon}_{r} \cos \left(\theta_{i}\right)+\sqrt{\varepsilon_{r}-\operatorname{sen}^{2}\left(\theta_{i}\right)}}
\end{aligned}
$$

onde a constante dielétrica complexa

$$
\bar{\varepsilon}_{\mathrm{r}=\varepsilon_{\mathrm{r}}}+\mathrm{j}\left(\frac{\mathrm{n}}{2 \pi}\right) \sigma \lambda
$$

Para campos difratados a direção dos raios que sofrem difração por uma quina condutora descreve um cone, cujo vértice é o ponto de difração.

Esse procedimento exige um novo lançamento de raios, porém, com a particularidade do ângulo " $\beta$ " ser constante e $\phi$ variar no espaço angular preenchido pelos raios $2 \pi-\Psi$, sendo $\Psi=2-\mathrm{n} \pi$, conforme a figura 6 .

Sobre a consideração que a quina é extendida ao infinito ao longo da direção $\mathrm{z}$, as componentes elétricas do campo difratado que são paralelas $\left(\mathrm{E}_{\beta 2}^{\mathrm{d}}\right) \mathrm{e}$ $\left(\mathrm{E}_{\alpha 2}^{\mathrm{d}}\right)$ perpendicular ao plano de difração são dadas por:

$$
\left[\begin{array}{c}
\mathrm{E}_{\beta 2}^{\mathrm{d}}(\mathrm{s} 2) \\
\mathrm{E}_{\alpha 2}^{\mathrm{d}}(\mathrm{s} 2)
\end{array}\right]=-\left[\begin{array}{cc}
\mathrm{D}_{\mathrm{s}} & 0 \\
0 & \mathrm{D}_{\mathrm{h}}
\end{array}\right]\left[\begin{array}{c}
\mathrm{E}_{\beta 1}^{\mathrm{i}}(\mathrm{D}) \\
\mathrm{E}_{\alpha 1}^{\mathrm{i}}(\mathrm{D})
\end{array}\right]
$$

$\mathrm{A}(\mathrm{s} 1, \mathrm{~s} 2) e^{-j k s 2}$

Onde $\mathrm{D}_{\mathrm{s}}$ e $\mathrm{D}_{\mathrm{h}}$ são os coeficientes de difração para a polarização "soft" e "hard", respectivamente.

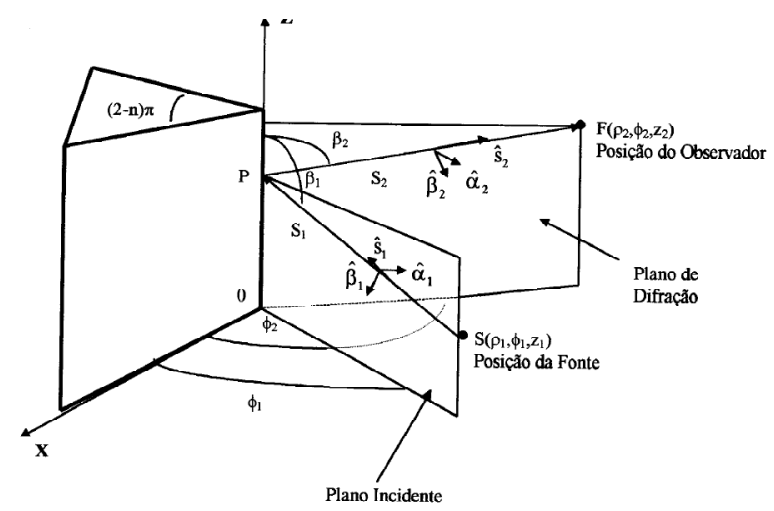

Figura 6- Geometria 3D de uma onda incidente oblíqua difratada numa cunha condutora onde:

$$
\begin{aligned}
& \mathrm{D}_{\mathrm{s} \cdot \mathrm{h}}=\frac{1}{\operatorname{sen} \beta_{1}}\left(\left\{\mathrm { d } ^ { + } ( \alpha ^ { - } ) \left(\mathrm{~F}\left[\mathrm{kLg}^{+}\left(\alpha^{-}\right)\right]+\mathrm{d}^{-}\left(\alpha^{-}\right)\right.\right.\right. \\
& \left(\mathrm{F}\left[\mathrm{kLg}^{-}\left(\alpha^{-}\right)\right]\right\} \pm \mathrm{d}^{+}\left(\alpha^{+}\right)\left(\mathrm{F}\left[\mathrm{kLg}^{+}\left(\alpha^{+}\right)\right]+\mathrm{d}^{-}\left(\alpha^{+}\right)\right. \\
& \left.\left(\mathrm{F}\left[\mathrm{kLg}^{-}\left(\alpha^{+}\right)\right]\right\}\right)
\end{aligned}
$$

Onde $\mathrm{D}_{\mathrm{s} \text { corresponde ao sinal negativo. }} \mathrm{d}^{ \pm}(\alpha)$ é definido por

$$
\mathrm{d}^{ \pm}(\alpha)=\frac{-e^{\mathrm{j} \pi / 4}}{2 \mathrm{n} \sqrt{2} 2 \pi \mathrm{k}} \cot \left[\frac{\pi \pm \alpha}{2 \mathrm{n}}\right]
$$

e F[x] é conhecida como a função de transição de Fresnel

$$
\mathrm{F}(\xi)=2 \mathrm{j} \sqrt{ } \xi e^{\mathrm{jx}} \int_{|\xi|}^{\infty} \exp \left(-\mathrm{j} \tau^{2}\right) \mathrm{d} \tau
$$

O argumento da função de transição é $\xi=\operatorname{kg}(\alpha)$, sendo k o número da onda e Lé o parâmetro distância que é dependente da forma da onda incidente e dado por:

$$
\mathrm{L}= \begin{cases}s 2 \sin ^{2} \beta_{1} & \text { onda plana incidente } \\ \frac{\rho_{1} \rho_{2}}{\rho_{1}+\rho_{2}} & \text { onda cilíndrica incidente } \\ \frac{\mathrm{s}_{1} \mathrm{~S}_{2}}{\mathrm{~s}_{1}+\mathrm{s}_{2}} \sin ^{2} \beta_{1} & \text { onda esférica incidente }\end{cases}
$$

A função $\mathrm{g}(\mathrm{a})$ é definida como $\mathrm{g}^{ \pm}(\alpha)=1+$ $\cos \left(\alpha-2 n N^{ \pm} \pi\right)$

Onde $\mathrm{N}^{+}=(1 / 2 \mathrm{n} \pi)$ e $\mathrm{N}^{-}=(1 / 2 \mathrm{n} \pi)\left(\alpha^{ \pm}-\pi\right)$, com $\mathrm{N}^{+}$ou $\mathrm{N}^{-}$sendo positivo ou negativo que melhor satisfaz a equação, $\alpha^{-}=\phi_{2}-\phi_{1}, \alpha^{+}=\phi_{2}+\phi_{1} \cdot \phi_{1}$ e $\phi_{2}$ são as componentes de $\left(\rho_{1}, \phi_{1}, z_{1}\right)$ e $\left(\rho_{2}, \phi_{2}, z_{2}\right)$, que são as posições da fonte do receptor, respectivamente, no sistema de coordenadas cilíndricas.

$\mathrm{O}$ fator de atenuação espacial $\mathrm{A}\left(\mathrm{s}^{\prime}, \mathrm{s}\right)$, que descreve como a intensidade do campo varia ao longo do raio difratado, é dado por

$$
A\left(\mathrm{~s} 1_{1}, \mathrm{~s}_{2}\right)= \begin{cases}\frac{1}{\sqrt{\mathrm{s}_{2}}} & \text { onda incidente cilíndrica } \\ \sqrt{\frac{\mathrm{s}_{2}}{\mathrm{~s}_{2}\left(\mathrm{~s}_{1}+\mathrm{s}_{2}\right)}} & \text { onda incidente esférica }\end{cases}
$$

No procedimento automatizado desenvolvido neste trabalho, leva-se em consideração os raios incidentes sobre as quinas verticais e horizontais (topo das construções), provocando o surgimento dos cones de difração, conforme mostrado na figura 7. 


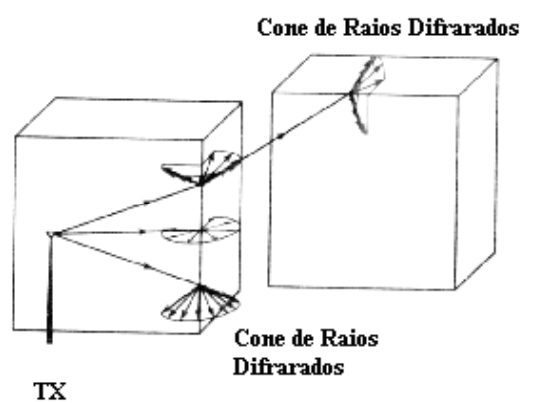

Figura 7- Cones de difração para quinas verticais e horizontais

\section{Considerações Finais}

Este software será desenvolvido na linguagem de programação Delphi utilizando seus recursos para computação gráfica. Envolvendo várias disciplinas como computação gráfica, eletromagnetismo, matemática, topografia, linguagem de programação e física. Este projeto é orientado aos alunos dos últimos anos dos cursos de graduação com o objetivo de desenvolver este procedimento automatizado com a finalidade de comercialização.

\section{Referências}

CHAMBERLIN K. A. and R. J. LUEBBERS , “An Evaluating of Longley-Rice and GTD Propagation Models “, IEEE Transactions on Antenas and Propagation, vol. 30, pp. 1093-1098, November 1982.

FARIA, ANTÔNIO AUGUSTO A. - Predição de Cobertura em Ambientes Indoor Utilizando Ray-Tracing Força Bruta Modificado, DECOM /FEE /UNICAMP. 1990.

KELLER, J.B. - Geometrical Theory of Diffraction, J. Opt. Soc. America, vol. 52, no. 2, pp 116-130, Fev. 1962.

RODRIGUES, M. E. DA COSTA - Técnicas de Traçado de Raios em Três Dimensões para Cálculo de Campos em Ambientes Interiores e Exteriores, Tese de Mestrado, PUC-RJ, Rio de Janeiro, 2000.

RUIZ-BOQUÉ S.; FERNADEZ; VILADERS M.; AUGUSTI, R .- Average Power Prediction in Microcells, Proceedings of the 44th IEEE Vehicular Technology Society Conference, 1994, pp.220-224.

Informações bibliográficas:

Conforme a NBR 6023:2002 da Associação Brasileira de Normas Técnicas (ABNT), este texto científico publicado em periódico eletrônico deve ser citado da seguinte forma:

FARIA JUNIOR, J. P. T.. Iluminação de Ambientes Tridimensionais Virtuais com Traçado de Raios. Cadernos UniFOA, Volta Redonda, ano II, n. 4, agosto. 2007. Disponível em: <http://www.unifoa.edu.br/pesquisa/caderno/edicao/04/11.pdf $>$ 